\title{
Multiple functional domains of human transcription factor IIB: distinct interactions with two general transcription factors and RNA polymerase II
}

\author{
Ilho Ha, ${ }^{1}$ Stefan Roberts, ${ }^{2}$ Edio Maldonado, ${ }^{1}$ Xiaoqing Sun, ${ }^{1}$ Leu-Ung Kim, ${ }^{1}$ Michael Green, ${ }^{2}$ \\ and Danny Reinberg ${ }^{1,3}$ \\ 'Department of Biochemistry, Robert Wood Johnson Medical School, University of Medicine and Dentistry of New Jersey, \\ Piscataway, New Jersey 08854-5635 USA ${ }_{i}{ }^{2}$ Program in Molecular Medicine, University of Massachusetts Medical Center, \\ Worcester, Massachusetts 01605 USA
}

Transcription factor IIB (TFIIB) plays a pivotal role in the formation of transcription-competent initiation complexes. TFIIB was found to interact with the TATA-binding protein, the small subunit of TFIIF, and RNA polymerase II. These interactions require distinct domains in TFIIB. Using the gel mobility-shift assay, it was found that the amino terminus of TFIIB was necessary for the formation of complexes containing RNA polymerase II and TFIIF, whereas the carboxy-terminal domain, which is composed of two imperfect direct repeats and includes a putative amphipathic $\alpha$-helix, was sufficient for the formation of complexes containing the TATA-binding protein and TFIIB (DB complex). Protein-protein interaction analyses demonstrate that the amphipathic $\alpha$-helix in TFIIB is important for the interaction with the TATA-binding protein. Specific residues mapping to the carboxyl terminus of the second direct repeat were found to be crucial for the interaction of TFIIB and RNA polymerase II. The interaction with the small subunit of TFIIF was mapped to the amino terminus of TFIIB, which includes a zinc finger.

[Key Words: TFIIB; TATA-binding protein; RNA polymerase II; interaction analyses]

Received February 2, 1993; revised version accepted April 20, 1993.

Identification of the protein factors that govern transcription initiation by RNA polymerase II has led to the delineation of two functional groups of transcription factors. One group, the general transcription factors (GTFs), catalyze basal transcription through the TATA and initiator DNA elements in a multiprotein complex with RNA polymerase II (RNAPII) (for review, see Weis and Reinberg 1992; Zawel and Reinberg 1992). The second group, the specific factors, is composed of DNA-binding proteins that bind independently of RNAPII to specific DNA sequences upstream of the TATA and initiator DNA elements (for review, see Johnson and McKnight 1989; Mitchell and Tjian 1989). The specific transcription factors are thought to participate directly in regulatory networks that mediate either stimulation or repression of basal transcription of specific genes. It is likely that the specific transcription factors exert their modulatory actions on basal transcription by interacting directly with the GTFs and RNAPII /for review, see

\footnotetext{
${ }^{3}$ Corresponding author.
}

Ptashne and Gann 1990). To date, seven human GTFs have been identified and purified extensively and four have been cloned (indicated by asterisks): TFIID ${ }^{\star}$, TFIIA, TFIIB ${ }^{*}$, TFIIF* ${ }^{*}$ TFIIE*, TFIIH, and TFIIJ.

The formation of transcription-competent complexes on class II promoters is a highly ordered process. In vitro complex formation is initiated on TATA-containing promoters by the association of TATA-binding protein (TBP, a subunit of TFIDD) (Pugh and Tjian 1990) to the TATA motif. The TBP-TATA complex provides a recognition site for the entry of TFIIB. TFIIB is essential for transcription from class II promoters. TFIIB is thought to participate in the formation of a stable preinitiation complex, as it is required to establish heparin-resistant transcription (Reinberg and Roeder 1987). Consistent with such a role, TFIIB recognizes the TBP-DNA complex and binds to it, generating the DB complex in the gel mobility-shift assay (Buratowski et al. 1989; Maldonado et al. 1990). Purified to homogeneity, the TFIIB polypeptide exhibited a molecular mass of $33 \mathrm{kD}$. The nucleotide sequence of the human TFIIB (hTFIIB) cDNA clone predicts an open reading frame encoding a polypeptide of 
Ha et al.

316 amino acids with a calculated molecular mass of $34.8 \mathrm{kD}$ (Ha et al. 1991; Malik et al. 1991). Also, cDNA clones encoding TFIIB activity have been isolated from Drosophila (Wampler and Kadonaga 1992; Yamashita et al. 1992), Xenopus (Hisatake et al. 1991), rat (Tsuboi et al. 1992), and yeast Saccharomyces cerevisiae (Pinto et al. 1992). The different cDNAs reveal motifs in TFIIB that could be potentially important for activity. A putative zinc finger is located at the amino terminus of the protein, and an imperfect repeated motif of 76 amino acids is present in the carboxy-terminal half of the protein. The presence of a structurally similar motif in TBP suggests that these repeats may be functionally important. In the $\sim 20$ amino acids separating the repeats in TFIIB, there is a region bearing some similarity to prokaryotic $\sigma$-factors. Also, nested between these repeats is an $\sim 15$ amino acid domain with the potential to form an amphipathic $\alpha$-helix containing hydrophobic residues along one side and charged basic residues along the other (Ha et al. 1991; Malik et al. 1991).

Recently, a model implicating TFIIB as being critical in mediating responses to upstream activators has emerged. Lin and Green (1991) demonstrated that the synthetic acidic activator Gal4-AH stimulates transcription by affecting TFIIB in the preinitiation complex. The acidic activator stimulates transcription levels by recruiting and/or maintaining TFIIB in the promoter region. Consistent with this model, it was shown that a direct and specific interaction occurs between native or recombinant TFIIB and the VP16 activating region (Lin et al. 1991). This interaction is important for activation, as a mutant VP16, which is unable to support activation, failed to interact with TFIIB.

To gain insight into the role of TFIIB during transcription, we have analyzed domains in TFIIB important for transcription. Our studies have indicated that TFIIB is composed of different domains that interact with the TBP, RNAPII, and RAP30, the small subunit of TFIIF.

\section{Results}

\section{TFIIB interacts with TBP, RNAPII and RAP3O}

Preinitiation complex formation studies, using the gel mobility-shift assay, have revealed that TFIIB enters into the transcription cycle after association of TBP with the TATA motif. These studies have also demonstrated that the TFIIB/TBP/TATA complex (DB complex) is necessary for TFIIF to escort RNAPII into the preinitiation complex. Such studies suggest that TFIIB will interact with TBP as well as with RNAPII and/or TFIIF. Thus, we analyzed which of these factors (TBP, TFIIF, RNAPII) interact with TFIIB. The interaction of TFIIB with TBP and TFIIF was analyzed using glutathionine $S$-transferase (GST) fusion proteins (GST-TBP and GST-RAP30), which were immobilized on glutathione-agarose beads. The interaction with RNAPII was analyzed by affinity chromatography on columns containing immobilized RNAPII. The ability of the different columns to retain TFIIB was monitored by Western blot using anti-TFIIB antibodies.
The results presented in Figure $1 \mathrm{~A}$ demonstrate that TFIIB interacts with TBP (lane 3), as well as with the RAP30 subunit of TFIIF (lane 5) and, as demonstrated previously, with the activator VP16 (lane 2). These interactions are specific, as TFIIB could not be retained on columns containing only GST (lane 1), or a fusion protein composed of GST and the species-specific amino terminus of human TBP (hTBP) (residues 1-165, $\Delta 166-$ 335) (lane 4). A similar analysis demonstrates an interaction of TFIIB with RNAPII, as TFIIB was retained by a column containing RNAPII and could be eluted with a $0.8 \mathrm{M} \mathrm{KCl}$ wash (Fig. 1B, lanes 6-9). This interaction appears specific, because when BSA was used as the ligand, all of the TFIIB was recovered in the flowthrough of the column (Fig. 1B, lanes 2-5). Thus, these results demonstrate specific interaction of TFIIB with three components of the transcription machinery, namely TBP, RAP30, and RNAPII.

The amphipathic $\alpha$-helix of TFIIB is necessary for interaction with $h T B P$

To analyze the domain in TFIIB necessary for interaction with TBP, a series of amino-terminal, carboxy-terminal, and in-frame internal deletion mutants were produced (see Fig. 2A). The mutant proteins were purified from Escherichia coli to $\sim 80 \%$ homogeneity (data not shown) and analyzed for their ability to interact with TBP in the

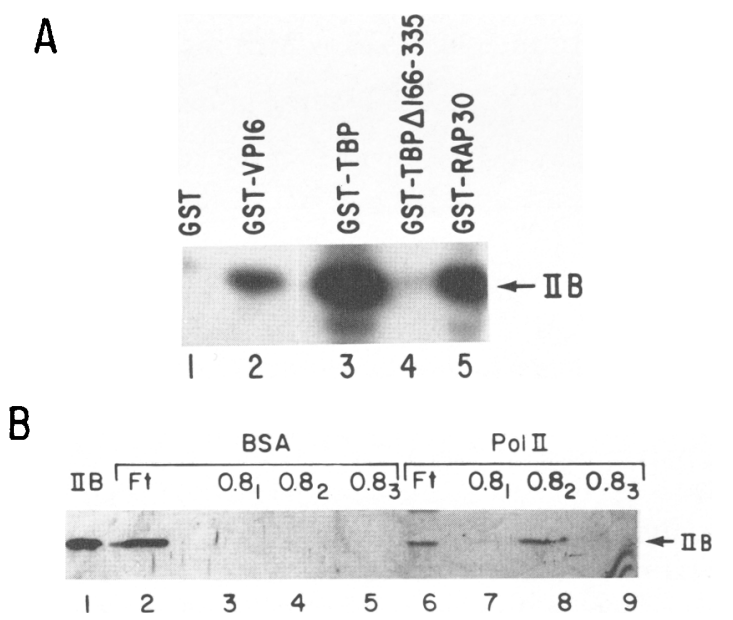

Figure 1. TFIIB interacts with TBP, RAP30, and RNAPII. (A) Bacterial lysate containing recombinant hTFIIB was incubated with $30 \mu \mathrm{l}$ of either GST, GST-VP16, GST-hTBP, GSThTBP $166-335$, or GST-RAP30 linked to glutathione-agarose beads for $1 \mathrm{hr}$ followed by extensive washing. The bound proteins were eluted in SDS-PAGE sample buffer, resolved by electrophoresis, and analyzed by Western blotting. $(B)$ Bacterial lysate $(200 \mu$ l) containing recombinant hTFIIB was incubated with $100 \mu \mathrm{l}$ of Affigel 10-RNAPII or Affigel 10-BSA. After washing the columns, the proteins were eluted with buffer $C$ containing $0.8 \mathrm{M} \mathrm{KCl}$, resolved by electrophoresis, and analyzed by Western blot. 


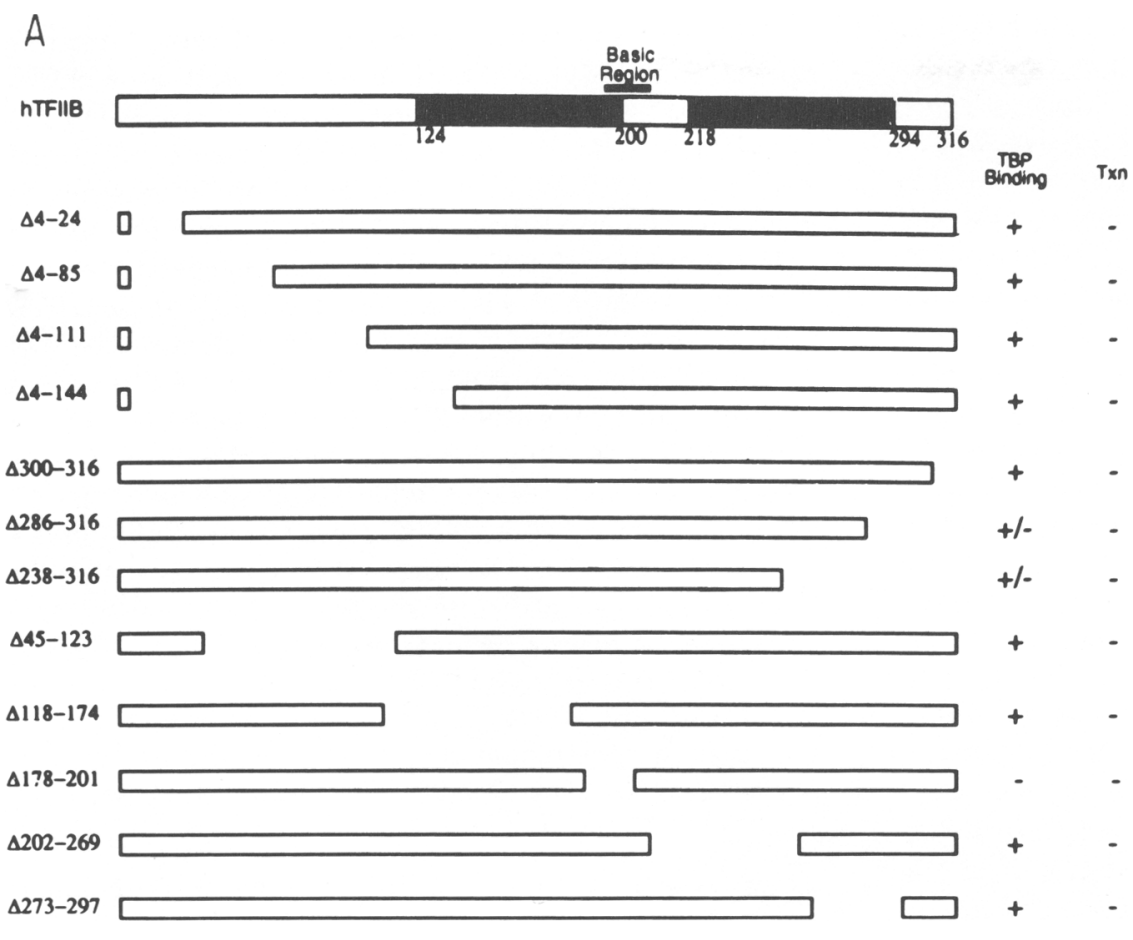

B

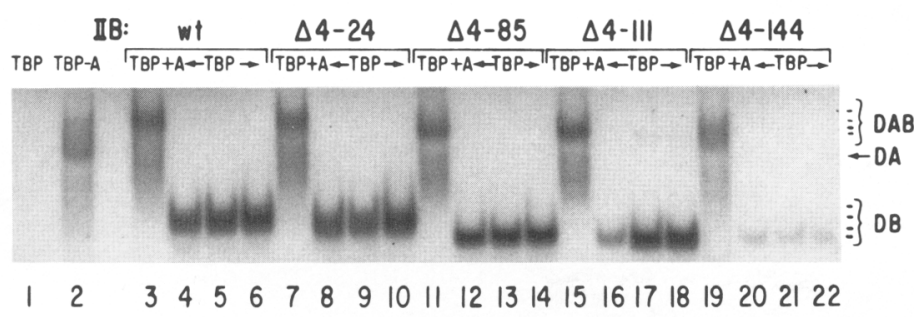

C

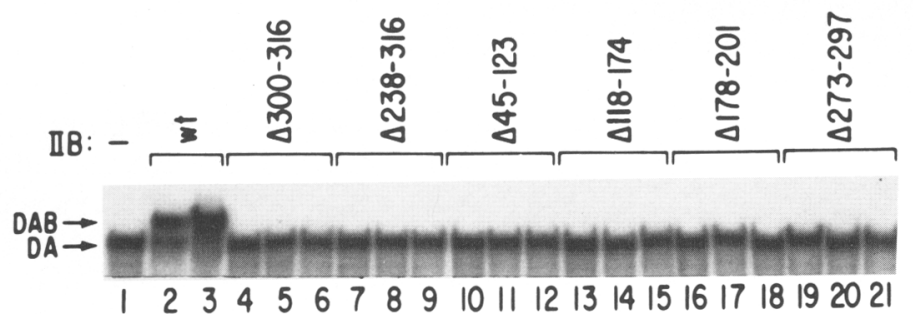

Figure 2. Mapping domains in TFIIB necessary for the formation of the $\mathrm{DB}$ and DAB complexes. $(A)$ Schematic representation of the different TFIIB mutant proteins. The symbols at the right denote the relative activity of the different TFIIB proteins. $(B, C)$ The ability of the different TFIIB proteins to form the $D B$ and $D A B$ complex was analyzed using the gel mobility-shift assay. The different complexes are indicated on the right $(B)$ and left $(C)$. The amount of TFIIB added was 10, 20, and $40 \mathrm{ng}$ for assays measuring the $\mathrm{DB}$ complex, and $20 \mathrm{ng}$ for assays measuring the DAB complex. Similar amounts were used in the analysis of $C$.

gel mobility-shift assay (formation of DB complex). This analysis demonstrates that the amino-terminal 111 residues of TFIIB are not required for formation of the DB complex (Fig. 2B). However, a mutant TFIIB protein including a deletion of the first 144 amino acids (TFIIB $\Delta 4$ 144), which removes 9 residues of the amino terminus of the first direct repeat, showed reduced activity (Fig. 2B, lanes 20-22). Because TBP binds weakly to the TATA motif, the formation of the DB complex depends on both the ability of TFIIB to interact with TBP as well as on the ability of TFIIB to stabilize the binding of TBP to the TATA motif; thus, the interaction of TFIIB with TBP was also monitored in the presence of TFIIA, which provides stable binding of TBP to the TATA motif (DA com- plex). Consistent with the above data, deletion of the first amino-terminal 111 amino acids did not affect formation of the DAB complex (Fig. 2B). However, under these conditions, TFIIB $\Delta 4-144$ was capable of producing the DAB complex (lane 19). The different mutants, although capable of interacting with the DA complex, were unable to participate in transcription (data not shown, see Fig 2A). Thus, the amino terminus of TFIIB is not necessary for the interaction with TBP but is required for basal transcription.

None of the carboxy-terminal or in-frame internal deletion mutants were capable of producing the $\mathrm{DAB}$ complex (Fig $2 \mathrm{C}$ ) and, consequently, were not able to participate in transcription (data not shown; see Fig 2A). The 
inability of the in-frame internal deletion TFIIB $\Delta 45-123$ to form the $\mathrm{DAB}$ complex was surprising because a deletion of the first 144 amino acids resulted in a polypeptide capable of producing the DAB complex (Fig. 2B). A possible interpretation of these observations is that this internal deletion may affect the structure of the protein rendering the DAB complex, containing TFIIB $\Delta 45-123$, unstable. Because TFIIB does not recognize any specific sequences on the promoter, the association of TFIIB with the DA complex results solely from an interaction with TBP; thus, we analyzed the domains in TFIIB necessary for the interaction with TBP in the absence of DNA.

E. coli extracts containing the different TFIIB mutants were analyzed for interaction with TBP as described in Figure 1A. Consistent with the results observed using the gel mobility-shift assay, this analysis revealed that the amino-terminal 144 amino acids are not important for the interaction with TBP (Fig. 3A). Similar analysis using carboxy-terminal truncations demonstrated that the last 16 amino acids of TFIIB were also not important for the interaction with TBP; however, further carboxy-

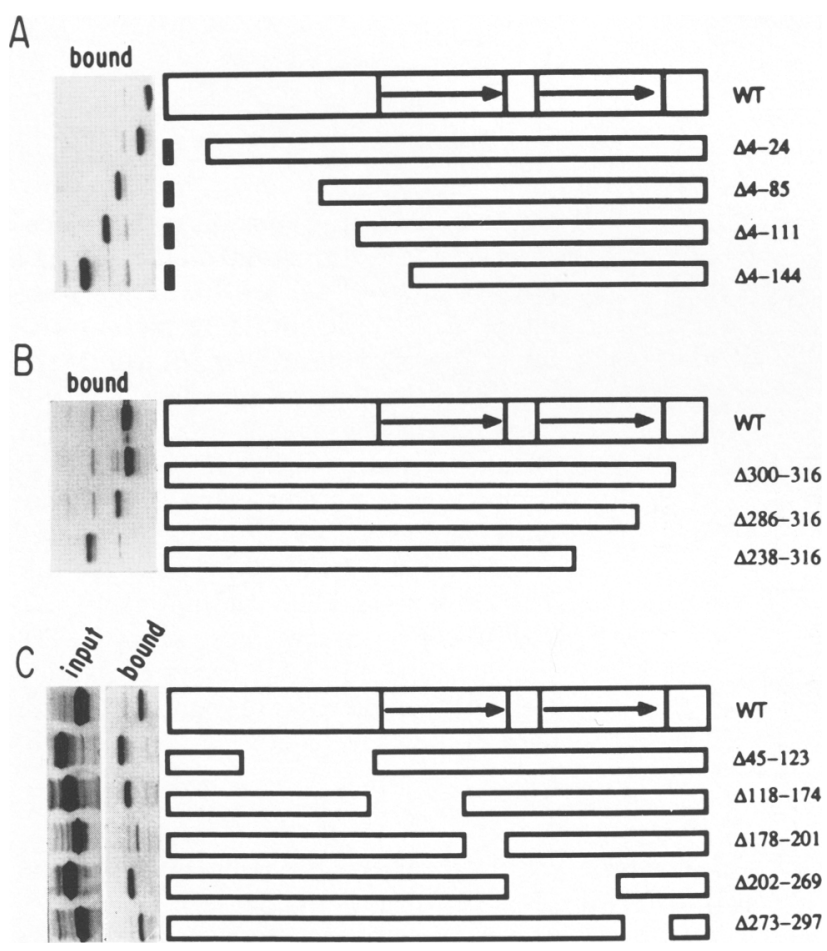

Figure 3. Interaction of TFIIB derivatives with immobilized GST-hTBP. Protein-binding assays were performed using GSThTBP and the different TFIIB mutant proteins. (A) Amino-terminal deletion; $(B)$ carboxy-terminal deletion; $(C)$ internal deletion. $E$. coli extracts containing each of the deletion proteins were incubated with hTBP-GST-agarose beads. After extensive washing, the bound proteins were loaded on SDS-Polyacrylamide gels and transferred to nitrocellulose. The blots were analyzed using anti-TFIIB antibody. (WT) Wild-type TFIIB. Each deletion is shown as a diagram. Input in $C$ denotes a Western blot showing amounts of the different TFIIB mutant proteins used in this analysis. terminal deletions reduced the interaction by $\sim 60 \%$ (Fig. $3 \mathrm{~B})$. Thus, the analyses with the amino-terminal and carboxy-terminal truncations demonstrated that the two direct repeats present in TFIIB are sufficient for efficient interaction with TBP. The analysis of in-frame internal deletions of TFIIB further delineated the residues necessary for interaction with TBP to amino acid residues 178-201, located at the carboxyl terminus of the first repeat; all other deletions were without effect (Fig. 3C, compare bound with input). Interestingly, amino acids 178-201, which drastically reduced the interaction with TBP when deleted, comprise a putative amphipathic $\alpha$-helix with basic and hydrophobic residues located on different sides of the helix (see $\mathrm{Ha}$ et al. 1991). Although these results clearly indicate that the putative amphipathic $\alpha$-helix is required for interaction with TBP, single and double substitutions of the basic residues did not preclude the ability of TFIIB to support basal transcription (Roberts et al. 1993). These combined results suggest that there is redundancy in the residues of the amphipathic $\alpha$-helix involved in the TBP interaction.

\section{Residues in hTBP important for interaction with TFIIB}

To define a motif in hTBP necessary for interaction with TFIIB, a series of carboxy-terminal (Fig. 4A) and aminoterminal (Fig. 4B) deletions were constructed. ${ }^{35} \mathrm{~S}-\mathrm{La}$ beled proteins were produced by in vitro transcription/ translation, and equal amounts of labeled proteins (input in Fig. 4, A and B) were incubated with GST or GSTTFIIB immobilized on glutathione-agarose beads. Analysis with the carboxy-terminal truncations (Fig. 4A) demonstrated that the second direct repeat of TBP could be removed almost entirely (residues 271-339) without affecting the interaction with TFIIB. A deletion removing the amino acids between the two direct repeats, as well as the second repeat (residues 221-339), reduced binding to TFIIB by $\sim 50 \%$. However, a carboxy-terminal deletion that included the last 20 amino acids of the first repeat (residues 201-339) prevented the interaction with TFIIB (Fig. 4A). Similar analysis with amino-terminal truncations demonstrated, as expected, that the speciesspecific amino terminus of hTBP was dispensable (Fig. 4B). Further amino-terminal deletions indicated that the first 194 amino acids, including almost entirely the first direct repeat, could also be removed without affecting binding to TFIIB (Fig. 4B). The amino- and carboxy-terminal truncations delineate a motif of 20 amino acids present at the carboxyl terminus of the first direct repeat, which appears important for the interaction with TFIIB. Thus, an in-frame internal deletion removing these 20 amino acids included in TBP $\Delta 169-220$ was analyzed for its ability to interact with TFIIB. Consistently, we found this mutant TBP protein to be unable to interact with TFIIB (Fig. 4C). Thus, the combined analysis of the amino- and carboxy-terminal truncations, together with the in-frame internal deletion mutant of hTBP (TBPA169-220), indicates that amino acids 201-221, located at the carboxyl terminus of the first direct repeat, are important for the interaction with TFIIB. 
RNAPII, TFIIB, protein interaction, and transcription

A
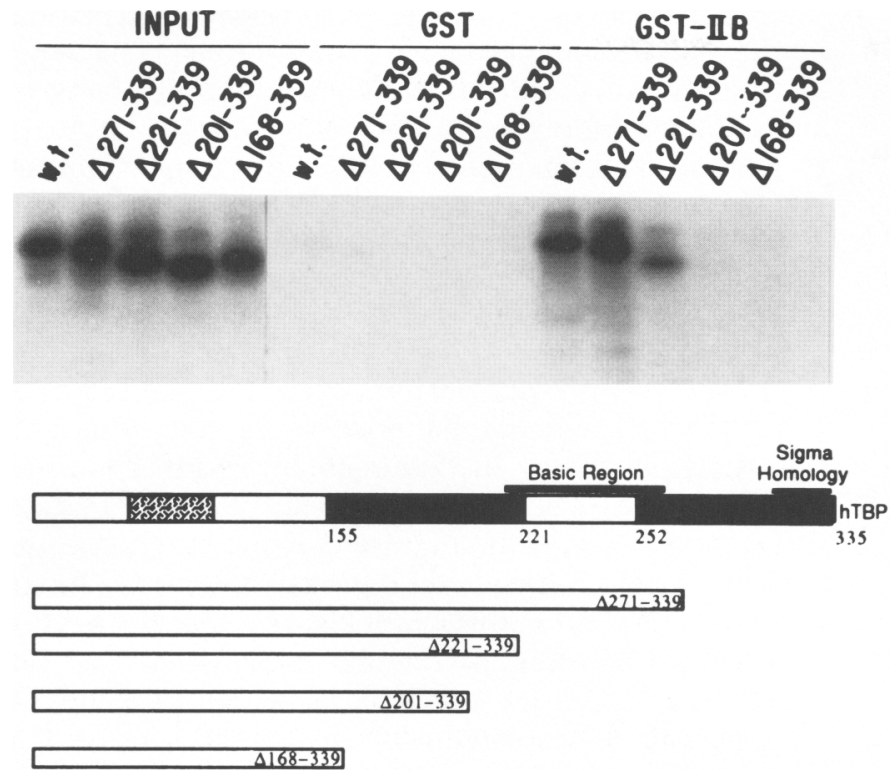

B
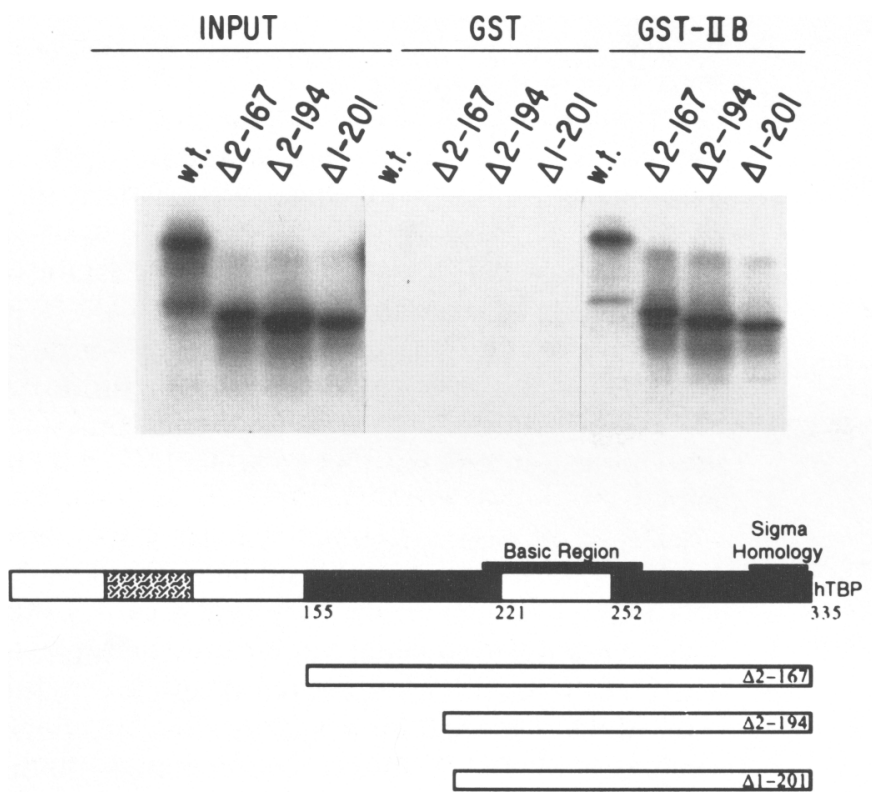

C
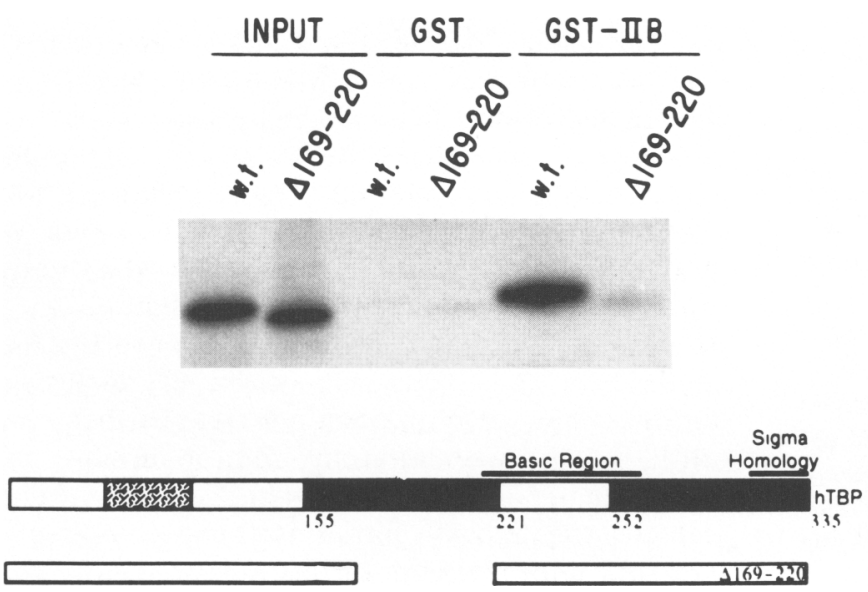
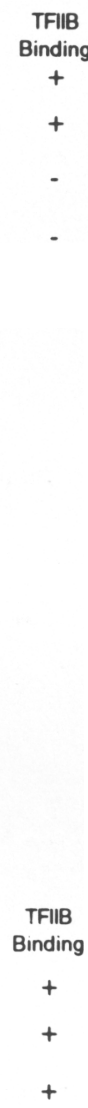

Figure 4. Interaction of hTBP derivatives with immobilized GST-TFIIB. $\left[{ }^{35}\right.$ S]Methionine-labeled $\mathrm{hTBP}$ and derivatives were produced by in vitro transcription/translation and equal amounts (Input) incubated with either $1 \mu \mathrm{g}$ of GST or $1 \mu \mathrm{g}$ GST-hTFIIB (GSTIIB) linked to glutathione-agarose beads for 1 hr. After extensive washing, the bound proteins were eluted in SDS-PAGE sample buffer, resolved by electrophoresis, and visualized by fluorography. A schematic diagram of the derivatives is shown below each panel. 
The amino terminus of TFIIB is necessary

for the association of RNAPII/IIF

with the preinitiation complex

The studies described in Figure 1B demonstrate a specific interaction between RNAPII and TFIIB. To analyze domains in TFIIB important for this interaction, the different deletion mutants of TFIIB (see Fig. 2A) were analyzed for the ability to support association of RNAPII with the promoter using the gel mobility-shift assay (DBPolF complex). In contrast to the results obtained analyzing interaction with TBP, the amino terminus of TFIIB was found to be important for the formation of the DBPolF complex, as the removal of the first 24 amino acids, which includes a putative zinc finger, resulted in a drastic decrease of the amount of complex formed (Fig. 5A, cf. lane 4 with lanes $6-8$ ). Further amino-terminal truncations, extending up to residue 111 , were without any additional effect (lanes 10-16). However, a deletion removing 33 more residues, extending to residues in the
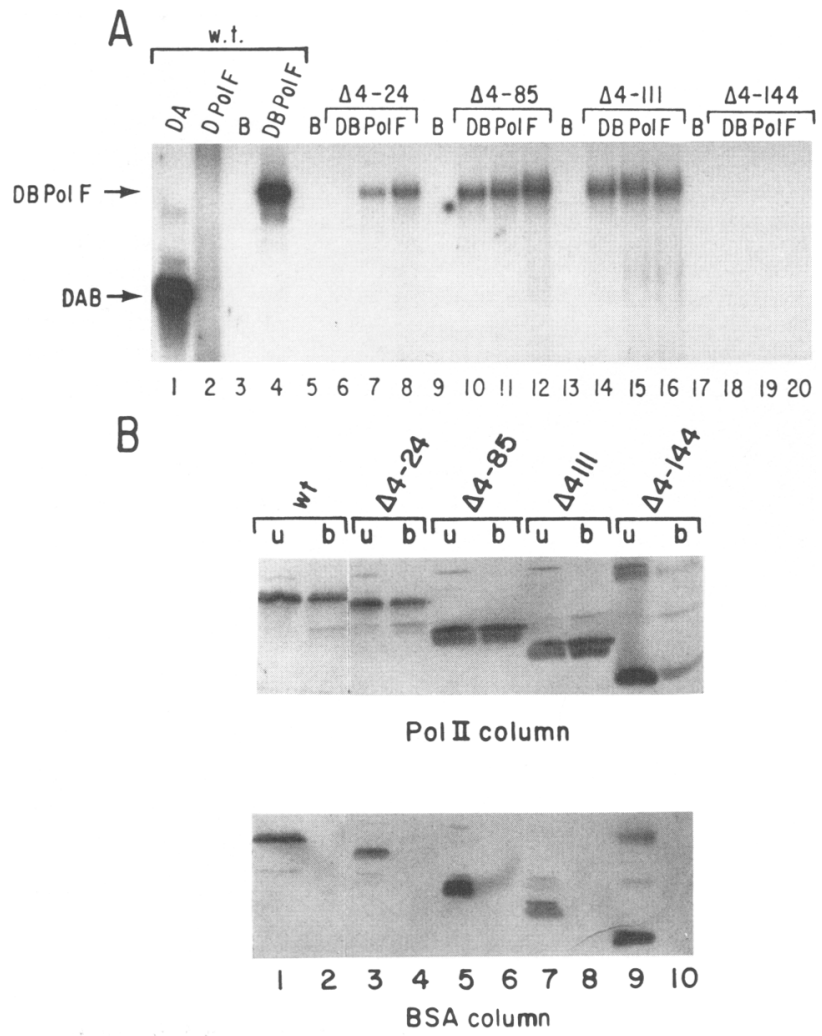

Figure 5. Interaction of TFIIB derivatives with RNAPII. $(A)$ Different TFIIB derivatives were analyzed for their ability to form the DBPolF complex using the gel mobility-shift assay. Complexes were formed as described and contained TBP (35 ng), RNAPII (40 ng), TFIIF ( $30 \mathrm{ng}$ ), and variable amounts (10, 20, and $40 \mathrm{ng}$ ) of the different TFIIB proteins. The amount of wild-type TFIIB protein used in this analysis was $20 \mathrm{ng}$. $(B)$ Interaction of TFIIB derivatives with immobilized RNAPII. Bacterial lysates $(200 \mu l)$ containing the different deletions mutants, as indicated at the top, were incubated with $100 \mu \mathrm{l}$ of Affigel 10-RNAPI (top) or Affigel 10-BSA, and analyzed as described in Fig. 1B. first direct repeat, completely abolished the formation of the DBPolF complex (lanes 18-20). Thus, TFIIB appears to have two independent domains: one involving residues amino-terminal to the first repeat, which participate in the formation of the DBPolF complex and thus interact with RNAPII and/or RAP30; and the amphipatic $\alpha$-helix, located at the carboxyl terminus of the first direct repeat, which is important for interaction with TBP.

\section{Residues in TFIIB necessary for interaction} with RNAPII

The studies described above demonstrate that amino acids amino-terminal to the first repeat of TFIIB were necessary for the formation of the DBPolF complex. Because the formation of this complex requires RNAPII and TFIIF, in addition to the DB complex, we analyzed whether the amino-terminal sequences of TFIIB were important for interaction with RNAPII and/or TFIIF. Amino-terminal truncations were analyzed for their ability to interact directly with RNAPII using RNAPII affinity chromatography, as described in Figure 1B. This analysis demonstrated that the first 111 amino acids of TFIIB were unnecessary for interaction with RNAPII (Fig. 5B, lanes 1-8). However, a deletion removing an additional 33 amino acids drastically reduced the interaction with RNAPII (lane 10). Thus, the analysis of complex formation (Fig. 5A), together with the analysis measuring direct interaction of TFIIB to RNAPII (Fig. 5B), suggested that the reduction in complex formation attributable to the removal of the first 24 amino acids of TFIIB (Fig. 5A, lanes 6-8) is likely to be a consequence of affecting the interaction between TFIIB and TFIIF (see below).

Analysis of carboxy-terminal truncations revealed that these residues were important for interaction with RNAPII, as the removal of the last 16 amino acids impairs the interaction (data not shown). Interestingly, when different in-frame internal deletion mutants (see Fig. 2A) were studied, it was found that the deletion of residues 202-269 resulted in increased binding to RNAPII by approximately twofold (data not shown; see below). However, the surrounding deletions, TFIIB $\Delta 178$ 201, which removed the putative amphipatic $\alpha$-helix, and TFIIB $\Delta 273-297$, as well as all of the other internal deletions, were defective in binding to RNAPII (data not shown; see below). We believed these results to be indicative of a large area in TFIIB necessary for interaction with RNAPII and that the different internal deletions affected the overall structure of TFIIB. However, we also thought that the increased binding observed with deletion TFIIB $\Delta 202-269$ may indicate residues important for interaction with RNAPII residing between residues 273 and 297. Thus, different amino acids were changed within residues 273 and 297, and the resulting proteins were analyzed for their ability to interact with RNAPII. Double point mutations changing arginine to glutamic acid at positions $286 / 290$ or $290 / 295$ decreased the interaction with RNAPII by approximately twofold (Fig. 
6A, lanes 10,12). Moreover, a similar triple substitution at positions 286,290 , and 295 abolished the interaction completely (Fig. 6A, lane 14). Single substitutions at these residues were without effect on the binding to RNAPII (Fig. 6A; data not shown). Interestingly the 290/ 295 and 286/290 double substitutions appear to drastically affect the structure of the proteins, as the mutant proteins migrated anomalously on polyacrylamide-SDS gels (Fig. 6B, lanes 6 and 7, respectively). This was analyzed further by subjecting one of the mutant proteins $(290 / 295)$ to mass spectroscopy analysis. These studies confirmed the substitutions and that the anomalous migration was the result of apparent changes in the structure of the mutant protein, as the mass of this protein was almost identical to the recombinant wild-type TFIIB protein (data not shown). The proteins with these double and triple substitutions did not affect the interaction with TBP (Fig. 6B). The interaction between the mutant TFIIB proteins and TBP remain specific, as TFIIB mutant proteins were unable to bind to the amino terminus species-specific residues of TBP $(\Delta 166-339$; see Fig. 4B) (Fig. $6 \mathrm{C})$. Consistent with the protein-protein interaction results, the triple point mutation was unable to form the DBPolF complex (Fig. 7A, lane 11) and was therefore transcriptionally inactive (Fig. 7B, lanes 2-5). Similarly, the double substitution changing arginine to glutamic acid at positions 286 and 290, which showed a reduced interaction with RNAPII, was also unable to form the DBPolF complex (Fig. 7A, lane 7) and, more importantly, was also unable to participate in transcription (Fig. 7B, lanes 10-13). However, a similar double substitution at positions 290/295, which resulted in a decreased interaction with RNAPII, also showed reduced ability to direct formation of the DBPolF complex (Fig. 7A, cf. lane 3 with 9 |, and was found to be capable of directing intermediate levels of transcription (Fig. 7B, cf. lanes 6-9 with 18-21, respectively). Interestingly, although the different TFIIB mutants proteins were found to be capable of interacting with TBP when analyzed using affinity chromatography, the proteins were unable to form the $\mathrm{DAB}$ complex when the interaction was analyzed using the gel mobility-shift assay (Fig. 7A). The most logical interpretation of these results is that these mutant proteins form the DAB complex, but with reduced stability, and thus the complex does not resist isolation by electrophoresis. This is consistent with the observation that the double substitutions appear to affect the structure of TFIIB, as anomalous migration on polyacrylamide-SDS gels was observed. The results presented above indicate that the arginines at positions 286, 290, and 295 are important for interaction with RNAPII.

\section{The amino terminus of TFIIB is required for interaction with RAP3O}

The studies measuring the formation of the DBPolF complex demonstrated that a deletion of the first 24 amino acids of TFIIB affects the formation of this complex (Fig. 6A). Nevertheless, studies measuring direct interaction of TFIIB with RNAPII or TBP demonstrated that the first 111 amino acids are dispensable for these interactions (Fig. 6B). Thus, these observations suggest that the amino terminus of TFIIB interacts with RAP30. To analyze this further, a series of mutant TFIIB proteins were analyzed for direct interaction with RAP30. These analyses demonstrated that the last 78 amino acids carboxy-terminal to TFIIB were dispensable (Fig. 8A, lane 6). More important, in-frame internal deletions removing residues 273-297, which eliminate interaction with RNAPII, or deletions of residues 202-269, were without effect in binding to RAP30 (lanes 11 and 10, respectively|. The carboxy-terminal truncations, together with the two in-frame internal deletions, demonstrate that residues between the two direct repeats, the entire second repeat, as well as amino acids carboxy-terminal to this repeat, could be removed without affecting the interaction with RAP30. However, the entire first repeat (lane 8), as well as residues amino-terminal to this repeat (lanes 4,5,7), affected the interaction with RAP30. These results are not attributable to variations on the amount of TFIIB protein bound to the resin (Fig. 8B) but to effects on the interaction between TFIIB and RAP30. Thus, sequences amino-terminal to the first direct repeat are important for interaction with RAP30.

\section{Discussion}

The studies described here demonstrate that TFIIB interacts with three components of the basal transcription machinery, namely the TBP, RNAPII, and RAP30. Using the gel mobility-shift assay, we found that the carboxyterminal domain, consisting of two imperfect 76-amino-
A

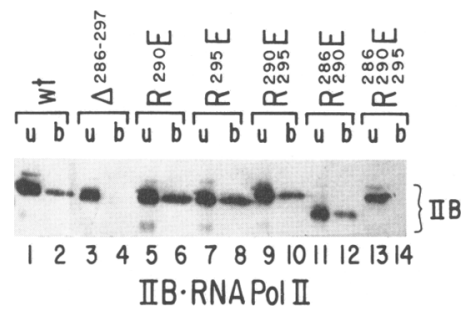

B

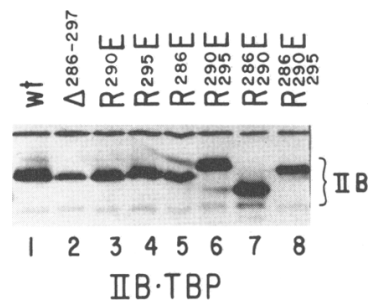

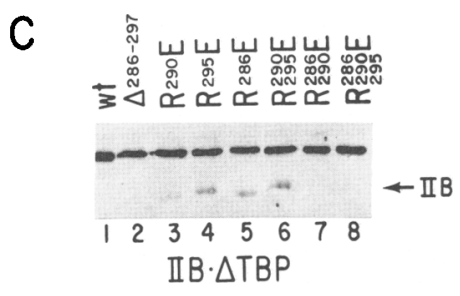

Figure 6. Double and triple amino acid substitution mutations in the carboxyl terminus of the second direct repeat of TFIIB decrease binding to RNAPII but not to TBP. Bacterial lysates $\{200 \mu l)$ containing the different point mutant proteins were incubated with 100 $\mu l$ of immobilized RNAPII $(A)$ GST-hTBP $(B)$, and GST-hTBP $\Delta 166-335(C)$, and analyzed as described in Fig. 1B. 
Ha et al.

A

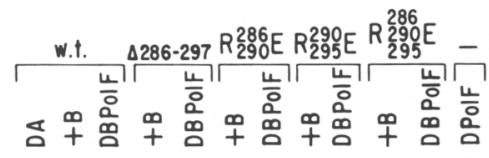
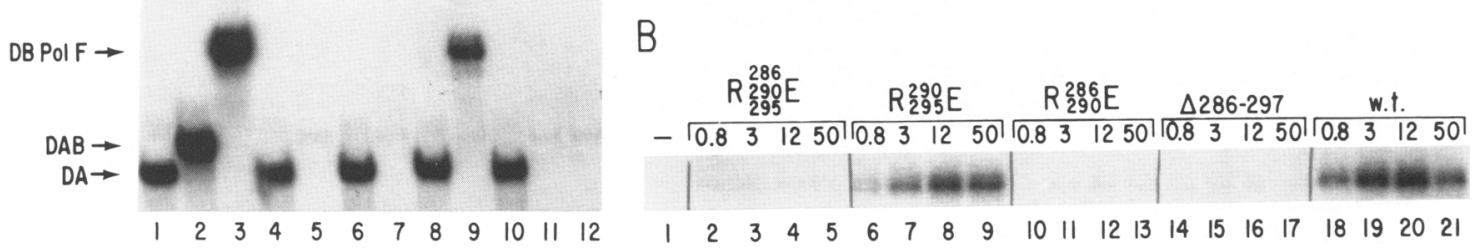

Figure 7. Transcriptional effect of TFIIB derivatives with double and triple substitutions in the RNAPII-binding motif. $(A \mid$ The different mutant proteins, as indicated at the top, were analyzed for their ability to form DAB and DBPolF complexes using the gel mobility-shift assay. Factors were as described in the legend to Fig. 6A. The amount of TFIIB protein added was $60 \mathrm{ng}$. (B) The different mutant proteins, as indicated at the top, were analyzed in transcription. Transcription assays were performed as described in Materials and methods. Proteins added were as follows: recombinant TFIIE (40 ng), TFIIF ( $30 \mathrm{ng}$ ), hTBP (70 ng), and TFIIB (indicated at the top). RNAPII (DEAE-5PW, $40 \mathrm{ng}$ ), TFIIH (phenyl-Superose, $1.8 \mu \mathrm{g}$ ), and TFII) (hydroxylapatite, $1.5 \mu \mathrm{g}$ ) were purified from HeLa cell nuclear extracts as described by Flores et al. (1991).

acid direct repeats and containing a putative amphipathic $\alpha$-helix is sufficient for interaction with the TBP, whereas the amino terminus of TFIIB was found necessary for the formation of complexes, including RNAPII. Because the gel mobility-shift assay does not allow for the isolation of unstable complexes and because TFIIB does not recognize any specific sequences in the promoter, we performed direct protein-protein interaction studies. This analysis led us to discover that TFIIB not only interacted with RAP30 through amino-terminal residues but that TFIIB also interacted with RNAPII. Moreover, the protein-protein interaction analysis allowed us to define different motifs in TFIIB mediating each interaction (see Fig. 9). We were able to map the interaction with TBP to a region at the carboxyl terminus end of the first direct repeat, which involved the putative amphipathic $\alpha$-helix. Similarly, the interaction with RNAPII was mapped specifically to residues located at the carboxyl terminus of the second direct repeat. Site-directed mutagenesis indicated that the basic residues located in the RNAPII-binding motif /residues 273-297) were important for the interaction with TFIIB. However, the net charge per se does not appear to be the main determinant in the interaction between TFIIB and RNAPII, as the substitution from arginine to glutamic acid at residues 290 and 295 had little effect on TFIIB

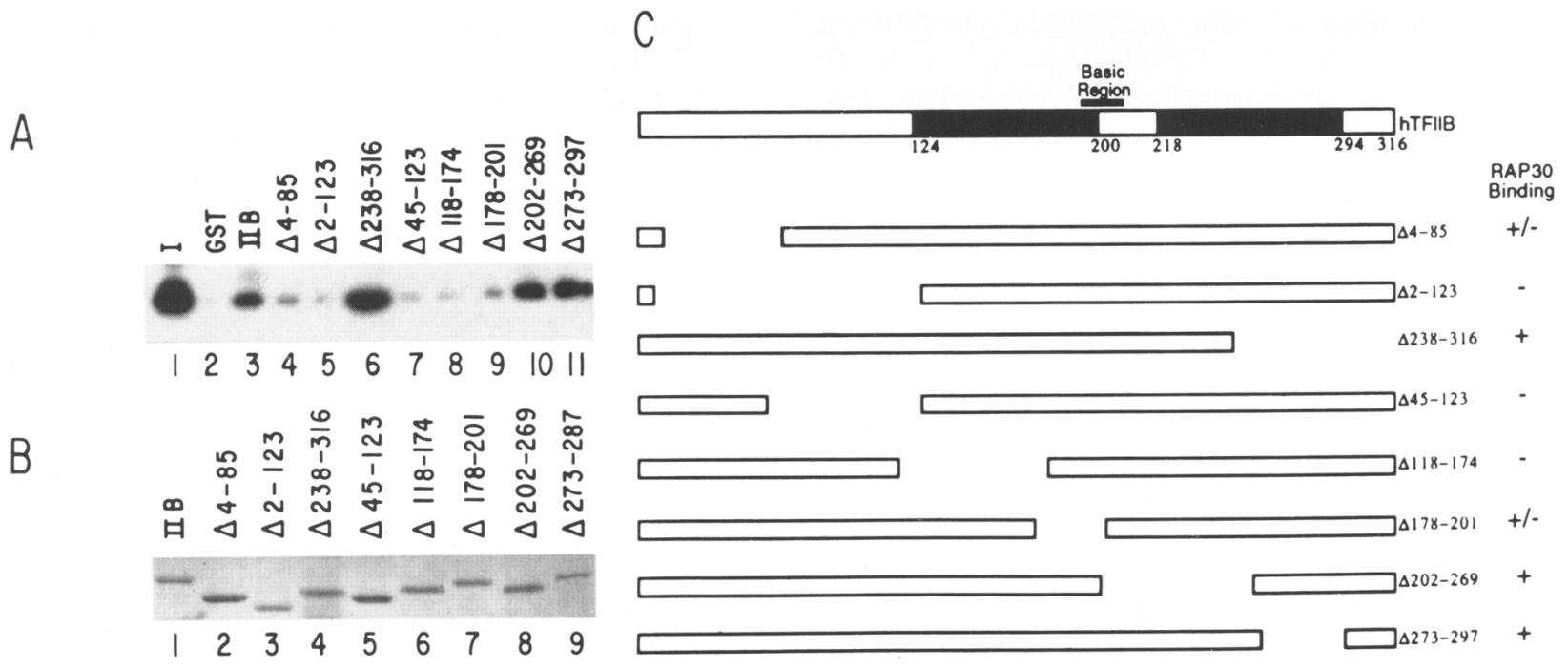

Figure 8. Specific interaction between RAP30 and immobilized TFIIB. (A) Purified HeLa cell TFIIF (I) was incubated with $1 \mu \mathrm{g}$ of either GST or the GST-TFIIB derivatives linked to glutathione-agarose beads for $1 \mathrm{hr}$. After extensive washing, the bound proteins were resolved by electrophoresis and visualized by Western blotting. $(B)$ Commassie blue staining of a protein gel containing an aliquot of the GST-TFIIB proteins used in the affinity chromatography analysis shown in $A$. $(C)$ A schematic diagram of the different GST-TFIIB derivatives. 


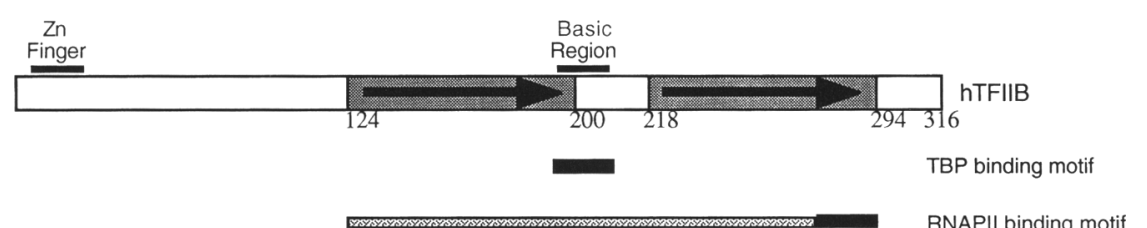

(2)
RNAPII binding motif RAP30 binding motif
Figure 9. Schematic representation of domains in TFIIB participating in interactions with TBP, RNAPII, and RAP30. For details, see Discussion. activity. It appears that the arginine residue at position 286 is important in this interaction, as a similar triple substitution including this residue, in addition to residues 290 and 295, which alone or together have little effect in TFIIB activity, resulted in a deleterious mutation. The importance of residue 286 in maintaining TFIIB activity is supported further by the finding that a similar double substitution at positions 286 and 290 was also deleterious for TFIIB activity. These residues appear specific in defining the interaction with RNAPII because mutations at positions 286, 290, and 295 were without effect in the interaction of TFIIB with TBP or RAP30.

The functions encoded in the highly structural carboxy-terminal domain of TFIIB appear to be independent of the amino terminus of the protein. Our studies demonstrate that the first amino-terminal 111 amino acids of TFIIB could be deleted without affecting the interaction with TBP or RNAPII. We found, however, that these residues were important for interaction with RAP30, the small subunit of TFIIF. The bipartite functionality of the amino and carboxyl terminus of TFIIB is further supported by findings demonstrating that the amphipathic $\alpha$-helix, which appears to maintain an ordered structure of the carboxy-terminal half of the protein and affects the interaction with TBP (Fig. 3) and RNAPII (data not shown), had no effect on the interaction with RAP30 (E. Maldonado, X. Sun, and D. Reinberg, unpubl.). The existence of two separable functional domains in TFIIB was noted also by Barberis et al. (1993), who isolated a 210amino-acid carboxy-terminal protease-resistant fragment of TFIIB that was capable of interacting with TBP but was unable to direct association of RNAPII/TFIIF with the preinitiation complex. Similar results were also obtained by Buratowski and Zhou (1993), who removed the first amino-terminal 40 amino acids of TFIIB and found the mutated protein capable of interacting with TBP but unable to produce the DBPolf complex. Buratowski and Zhou extended these studies and suggested that a putative zinc finger, present in the first aminoterminal 20 amino acids of TFIIB, may participate in the interaction with RNAPII/RAP30. The existence of two functional domains in TFIIB is supported further by the studies of Hisatake et al. (1993), who by using small deletions mapped the TBP-binding domain to the two imperfect direct repeats and the RNAPII/TFIIF-binding site to residues located at the amino terminus of TFIIB.

Interestingly, our previous studies have demonstrated an absolute requirement for TFIIF during the association of RNAPII with the DB complex (formation of DBPolF complex) (Flores et al. 1991). Nevertheless, in the studies presented here, we found that the DBPolF complex could be formed with a mutated TFIIB protein that removes the RAP30-binding motif /the amino-terminal 111 amino acids|, but at reduced levels. Under these conditions it is likely that RAP30 was brought into the complex by interacting with RNAPII. This result indicates that a single factor can interact with more than one component of the basal transcription machinery. This is supported further by our findings demonstrating the interaction between RNAPII and TFIIB, previous studies showing an interaction between TBP and the heptapeptides present at the carboxyl end of the largest subunit of RNAPII (Usheva et al. 1992), and studies demonstrating the interaction of TFIIF (RAP30) with RNAPII (Flores et al. 1989; McCracken and Greenblatt 1991). The redundancy of protein-protein interactions among the GTFs may provide the bases to explain the findings demonstrating that the formation of the preinitiation complex is highly cooperative and appears to be of profound importance during formation of transcription-competent complexes on TATA-less promoters /Weis and Reinberg 1992).

It is important to note the overall apparent similarity between TFIIB and the TBP. Both polypeptides contain functionally important direct repeats at their carboxyterminal ends separated by 20-30 amino acids. The residues separating these imperfect repeats contain basic amino acids. The evolutionary conserved direct repeats in TBP are important for the association of the protein with DNA (Yamamoto et al. 1992). Also, our analysis mapped the TFIIB-binding motif in TBP to 20 amino acids located at the carboxyl terminus of the first repeat, imbedded in elements apparently important for DNA recognition (Nikolov et al. 1992). Similarly, the TBPbinding domain in TFIIB was mapped to the carboxyl terminus of the first repeat. The carboxyl terminus of the second repeat in TBP contains a motif with sequence homology to $\sigma$-factors (Horikoshi et al. 1989). In TFIIB, the carboxyl terminus of the second repeat contains the RNAPII-binding domain. TBP was found to be an essential component of the three RNA polymerase transcription systems (for review, see Sharp 1992; White and Jackson 1992). TFIIB, on the other hand, is specific for transcription by RNAPII; however, a factor [BRF1 (Colbert and Hahn 1992), TDS4 (Buratowski and Zhou 1992), PCF4 (Lopez-de-Leon et al. 1992)] with substantial homology to TFIIB, containing a putative zinc finger and two direct repeats, was found to be essential for transcription mediated by RNAPIII. This, together with the finding of a TFIIB homolog in the archaebacterium Py. 
rococcus woesei (Ouzounis and Sanders 1992), indicates that the role of TFIIB in transcription was set early during evolution before the divergence of the eukaryotic RNA polymerases. Both polypeptides, TBP and TFIIB, contain an amino terminus that can be removed without affecting the formation of the TFIIB/TBP/DNA complex. However, although the sequences at the amino terminus of TBP are not conserved throughout evolution and are not essential for basal transcription, the amino terminus of TFIIB is conserved throughout evolution and is essential for basal transcription. Our observations demonstrating that TFIIB contains domains mediating interaction with the TBP, TFIIF, and RNAPII indicate that TFIIB may serve as a bridge between promoterbound TBP and RNAPII. This function of TFIIB supports the fact that its activity must be subjected to tight regulation, which is in agreement with the finding that TFIIB is a target for acidic activators (Lin and Green 1991; Roberts et al. 1993).

\section{Materials and methods \\ Construction of deletion mutations in the hTFIIB gene}

For amino-terminal deletion mutants, the following oligonucleotides were designed as amino-terminal primers for PCR: $5^{\prime}$ dGCTAGCGAGGACTACAGAGCCG-3' ( $(44-24), 5^{\prime}$-dGCTAGCAAGGGCACAGGAGCTGC-3' ( $\triangle 4-85), 5^{\prime}$-dGCTAGCCGGGCAATGATGAATGC-3' $(\Delta 4-111)$, and 5'-dGCTAGCGTATATGAACAGAArAGC-3' $(\Delta 4-144)$ (the underlined nucleotides indicate an $N$ heI recognition site). The carboxy-terminal primer (5'-dCGGGATCCGACGTTAGCTGCCTC-3'; the underlined nucleotides represent a $B a m H I$ site) was synthesized. After PCR and restriction digestion with NheI and BamHI, the fragments were inserted into pETlla vector (Novagen, Inc.). The carboxy-terminal truncation $\Delta 300-316$ was constructed as follows: Plasmid phIIB was digested with BgIII (amino acid 299) and BamHI (located in the pET vector) and ligated. The resulting construct produced a TFIIB protein containing 18 extra amino acids (AANKARKEAELAAATAEQ). The carboxy-terminal truncation $\triangle 286-316$ was generated by inserting a stop codon (TAA) at amino acid 286 . The $\Delta 238-316$ carboxy-terminal truncation was produced by PCR; a pair of oligonucleotides (amino-terminal, 5'-dCGTGGCTAGCACCAGCCGTTTGGATGC- $3^{\prime}$, and carboxy-terminal, 5'-dGGATCCTAACGGGCTATATGTGTAGC-3'; the underlined nucleotides represent the NheI and BamHI sites) was used. For in-frame internal deletions, different restriction enzyme sites were used: NciI-NcoI

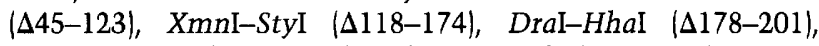

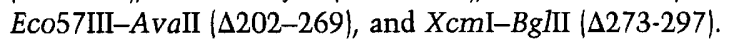

\section{Construction of point mutants in TFIIB}

A NheI-BamHI fragment from phIIB (Ha et al. 1991) was cloned into the NheI-BamHI sites of pBluescript SKII (Stratagene), and single-stranded DNA was produced. Oligonucleotides encoding the indicated mutations were used for site-directed mutagenesis using a kit from Amersham and according to the instructions of the supplier. Mutations were confirmed by dideoxy sequencing, and the mutated open reading frame was excised from pBluescript SKII and subcloned into the NheI-BamHI sites of pETIla (Novagen).

\section{Mutagenesis of hTBP}

The entire coding sequence of hTBP was cloned into pGEM- 3 by PCR, and mRNA was produced by transcription with SP6 RNA polymerase. The mRNA was translated either in rabbit reticulocyte lysate or wheat germ extract (Promega) in the presence of $\left[{ }^{35}\right.$ S]methionine. Carboxy-terminal truncations were produced by cleavage of pGEM-hTBP with StuI ( $\Delta 271-339)$, ApaLI ( $\Delta 221-$ $339), B s p H I(\Delta 201-339)$ or $S s p I(\Delta 168-339)$, followed by transcription and translation. Amino-terminal truncations were made by cloning the appropriate restriction fragments, or PCR products, in-frame to an ATG initiation codon in pGEM-3.

\section{Purification of different TFIIB proteins}

E. coli strain BL21 (DE3; Novagene, Inc.) containing phIIB or mutant plasmids was cultured in LB media supplemented with ampicillin $(100 \mu \mathrm{g} / \mathrm{ml})$ at $37^{\circ} \mathrm{C}$. Cells were induced with IPTG $(0.4 \mathrm{mM})$ when an $\mathrm{OD}_{600}$ of 0.6 was reached. After $2 \mathrm{hr}$, cells were harvested by centrifugation at $4000 \mathrm{~g}$ for $10 \mathrm{~min}$. The pellet was resuspended in $1 / 20$ of the original culture volume using buffer $\mathrm{C}$ containing $0.1 \mathrm{M} \mathrm{KCl}$ and stored at $-70^{\circ} \mathrm{C}$. The frozen cells were thawed and broken by sonication on ice. Cell debris was removed by centrifugation at $6000 \mathrm{~g}$ for $15 \mathrm{~min}$, and the supernatant was loaded onto phosphocellulose columns $15 \mathrm{mg}$ of protein $/ \mathrm{ml} \mathrm{resin}$, which were equilibrated with buffer $\mathrm{C}[20$ $\mathrm{mM}$ Tris $-\mathrm{HCl}$ at $\mathrm{pH} 7.9$ at $4^{\circ} \mathrm{C}, 20 \%$ (vol/vol) glycerol, $10 \mathrm{mM}$ $\beta$-mercaptoethanol, $0.1 \mathrm{mM}$ EDTA] containing $0.1 \mathrm{~m} \mathrm{KCl}$. Proteins were eluted from the column using buffer $\mathrm{C}$ containing 0.8 $\mathrm{M} \mathrm{KCl}$ as described by Ha et al. (1991).

\section{Protein-binding assays using GST fusion proteins}

The GST fusion proteins were constructed by inserting the open reading frame of the different proteins by PCR into PGEX2T (Pharmacia). The fusion proteins were expressed and columns were prepared as described by Lin and Green (1991). Approximately $30 \mu$ l of glutathione-agarose beads containing the fusion protein was incubated at $4^{\circ} \mathrm{C}$ in $0.6 \mathrm{ml}$ of buffer containing 100 $\mathrm{mM} \mathrm{KCl}, 40 \mathrm{~mm}$ HEPES (pH 7.5), $5 \mathrm{~mm} \mathrm{MgCl}_{2}, 0.2 \mathrm{~mm}$ EDTA, $1 \mathrm{mM}$ DTT, $0.5 \mathrm{mM}$ PMSF, and $0.5 \% \mathrm{NP}-40$. Equal amounts of either bacterial lysate or in vitro-translated protein were added, and binding was allowed to proceed for $1 \mathrm{hr}$. The beads were washed five times with the same buffer and the bound proteins eluted with $50 \mu \mathrm{l}$ of SDS-PAGE loading buffer and were resolved by electrophoresis. In vitro-translated proteins were visualized by fluorography. Bacterially produced proteins were visualized by Western blotting.

\section{RNAPII affinity chromatography}

RNAPII was purified as described by Lu et al. 1991. The DEAE$5 \mathrm{PW}$ fraction $(0.4 \mathrm{mg} / \mathrm{ml})$ was dialyzed against coupling buffer (100 mM HEPES at pH 7.9, 0.1 mm EDTA, $500 \mathrm{~mm} \mathrm{NaCl}, 10 \%$ glycerol) and incubated with Affigel 10 (Bio-Rad) at a ratio of 2 $\mathrm{mg} / \mathrm{ml}$ of resin, according to the manufacturer's instructions. After coupling, the resin was washed extensively with buffer C containing $0.1 \mathrm{M} \mathrm{KCl}$. Affinity columns were prepared by placing $100 \mu \mathrm{l}$ of resin into an econo column (Bio-Rad), which was incubated at $4^{\circ} \mathrm{C}$ for $30 \mathrm{~min}$ with $200 \mu \mathrm{l}$ of crude supernatant preapared as described in the previous section. The columns were washed extensively with at least $4 \mathrm{ml}$ of buffer $C$ containing $0.1 \mathrm{M} \mathrm{KCl}$, and the bound proteins were eluted with 3 column volumes of buffer $C$ containing $0.8 \mathrm{M} \mathrm{KCl}$. An aliquot $(5 \mu \mathrm{l})$ of the flowthrough and bound fractions was separated by SDSPAGE and analyzed by Western blot using anti-TFIIB antibod- 
ies. As control, BSA columns were used under identical circumstances.

\section{Other methods}

Transcription factors were purified as described by Flores et al. (1991). RNAPII was purified as described by Lu et al. (1991). DNA-binding and transcription assays were performed as described by Maldonado et al. (1990).

\section{Acknowledgments}

We thank Dr. A. Berk for the gift of the TBP $\Delta 169-220$ expression vector, Dr. W. Lane for performing the mass spectroscopy analysis, and Dr. N. Greenfield for performing CD analysis on different TFIIB protein mutants. We also thank Drs. A. Barberis, M. Ptashne, S. Buratowski, and R. G. Roeder for communicating results before publication. We are grateful to R. Drapkin and L. Zawel for active discussions. This work was supported by grants from the National Institutes of Health to D.R. and M.R.G. D.R. is the recipient of an American Cancer Society Faculty Research Award.

The publication costs of this article were defrayed in part by payment of page charges. This article must therefore be hereby marked "advertisement" in accordance with 18 USC section 1734 solely to indicate this fact.

\section{References}

Barberis, A., C.W. Miller, S.C. Harrison, and M. Ptashne. 1993. Delineation of two functional regions of TFIIB. Proc. Natl. Acad. Sci. (in press).

Buratowski, S. and H. Zhou. 1992. A suppressor of TBP mutations encodes and RNA polymerase III transcription factor with homology to TFIIB. Cell 71: 221-230.

- 1993. Functional domains of transcription factor IIB. Proc. Natl. Acad. Sci. (in press).

Buratowski, S., S. Hahn, P.A. Sharp, and L. Guarante. 1989. Five intermediate complexes in transcription initiation by RNA polymerase II. Cell 56: 549-561.

Colbert, T. and S. Hahn. 1992. A yeast TFIIB-related factor involved in RNA polymerase III transcription. Genes \& Dev. 6: $1940-1949$.

Flores, O., E. Maldonado, and D. Reinberg. 1989. Factors involved in specific transcription by mammalian RNA polymerase II. Factors IIE and IIF independent interact with RNA polymerase II. J. Biol. Chem. 264: 8913-8921.

Flores, O., H. Lu, M. Killeen, J. Greenblatt, Z.F. Burton, and D. Reinberg. 1991. The small subunit of transcription factor IIF recruits RNA polymerase II into the preinitiation complex. Proc. Natl. Acad. Sci. 88: 9999-10003.

Ha, I., W. Lane, and D. Reinberg. 1991. Cloning of a human gene encoding the general transcription factor IIB. Nature 352: 689-695.

Hisatake, K., S. Malik, R.G. Roeder, and M. Horikoshi. 1991. Conserved structural motifs between Xenopus and human TFIIB. Nucleic Acids Res. 19: 6639.

Hisatake, K., R.G. Roeder, and M. Horikoshi. 1993. Functional dissection of TFIIB domains required for TFIIB.TFIID. promoter complex formation and basal transcription activity. Nature (in press).

Horikoshi, M., C. Wang, H. Fuji, J. Cromlish, P.A. Weil, and R.G. Roeder. 1989. Cloning and structure of a yeast gene encoding a general transcription initiation factor TFIID that binds to the TATA-box. Nature 346: 387-390.
Johnson, P. and S. McKnight. 1989. Eukaryotic transcription regulatory proteins. Annu. Rev. Biochem. 58: 799-839.

Lopez-de-Leon, A., M. Librizzi, K. Puglia, and I.M. Willis. 1992. PCF4 encodes an RNA polymerase III transcription factor with homology to TFIIB. Cell 71: 211-220.

Lin, Y.S. and M.R. Green. 1991. Mechanism of action of an acidic transcriptional activator in vitro. Cell 64: 971-981.

Lin, Y.S., I. Ha, E. Maldonado, D. Reinberg, and M.R. Green. 1991. Binding of general transcription factor TFIIB to an acidic activating region. Nature 353: 569-571.

Lu, H., O. Flores, R. Weinmann, and D. Reinberg. 1991. The non-phosphorylated form of RNA polymerase II preferentially associates with the preinitiation complex. Proc. Natl. Acad. Sci. 88: 10004-10008.

Maldonado, E. I. Ha, P. Cortes, L. Weis, and D. Reinberg. 1990. Role of transcription factors IIA, IID, and IIB during formation of a transcription-competent complex. Mol. Cell. Biol. 10: 6335-6347.

Malik, S., K. Hisatake, H. Sumimoto, M. Horikoshi, and R.G. Roeder. 1991. Sequence of general transcription factor TFIIB and relationships to other initiation factors. Proc. Natl. Acad. Sci. 88: 9553-9557.

McCracken, S. and J. Greenblatt. 1991. Related RNA polymerase-binding regions in human RAP30/74 and Escherichia coli s70. Science 253: 900-902.

Mitchell, P.J. and R. Tjian. 1989. Transcriptional regulation in mammalian cells by sequence-specific DNA-binding proteins. Science 245: 371-378.

Nikolov, D.B, S.-H. Hu, J. Lin, A. Gasch, A. Hoffmann, M. Horikoshi, N.-H. Chua, R.G. Roeder, and S.K. Burley. 1992. Crystal structure of TFIID. TATA box binding protein. Nature 360: $40-46$.

Ouzounis, C. and C. Sander. 1992. TFIIB, and evolutionary link between the transcription machineries of archaebacteria and eukaryotes. Cell 71: 189-190.

Pinto, I., D.E. Ware, and M. Hampsey. 1992. The yeast SUA 7 gene encodes a homologue of human transcription factor TFIIB and is required for normal start site selection in vivo. Cell 68: 977-988.

Ptashne M. and A. Gann. 1990. Activators and targets. Nature 346: 329-331.

Pugh, F., B., and Tjian, R. 1990. Mechanism of transcriptional activation by Sp1: Evidence for coactivators. Cell 61: 11871197.

Reinberg, D. and R.G. Roeder. 1987. Factors involved in specific transcription by RNA polymerase II. Purification and functional analysis of initiation factors IIB and IIE. J. Biol. Chem. 262: 3310-3321.

Roberts, S.G.E., I. Ha, E. Maldonado, D. Reinberg, and M. Green. 1993. Interaction between an acidic activator and the general transcription factor IIB is required for transcriptional activation. Nature (in press).

Sharp, P.A. 1992. TATA-binding protein is a classless factor. Cell 68: 819-821.

Tsuboi, A., K. Conger, K.P. Garret, R.C. Conaway, J.W. Conaway, and N. Arai. 1992. RNA polymerase II initiation factor alpha from rat liver is almost identical to TFIIB. Nucleic Acids Res. 20: 3250

Usheva, A., E. Maldonado, A. Goldring, H. Lu, C. Houbavi, D. Reinberg, and Y. Aloni. 1992. Specific interaction between the non phosphorylated form of RNA polymerase II and the TATA binding protein. Cell 69: 871-881

Wampler, S.L. and J.T. Kadonaga. 1992. Functional analysis of Drosophila transcription factor IIB. Genes \& Dev. 6: 15421552.

Weis, L. and D. Reinberg. 1992. Transcription by RNA polymer- 
Ha et al.

ase II: Initiator-directed formation of transcription-competent complexes. FASEB I. 6: 3300-3309.

White, R. and S. Jackson. 1992. The TATA-binding protein: A central role in transcription by RNA polymerase I, II, and III. Trends Genet. 8: 284-288.

Yamamoto, T., M. Horikoshi, J. Wang, S. Hasegawa, P.A. Weil, and R.G. Roeder. 1992. A bipartite DNA binding domain composed of direct repeats in the TATA box binding factor TFIID. Proc. Natl. Acad. Sci. 89: 2844-2848.

Yamashita, S., K. Wade, M. Horikoshi, D. Gong, T. Kokubo, K. Hisatake, N. Yokotani, S. Malik, R.G. Roeder, and Y. Nakatani. 1992. Isolation and characterization of a CDNA encoding Drosophila transcription factor TFIIB. Proc. Natl. Acad. Sci. 89: 2839-2843.

Zawel, L. and D. Reinberg. 1992. Advances in RNA polymerase II transcription. Curr. Opin. Cell. Biol. 4: 488-495. 


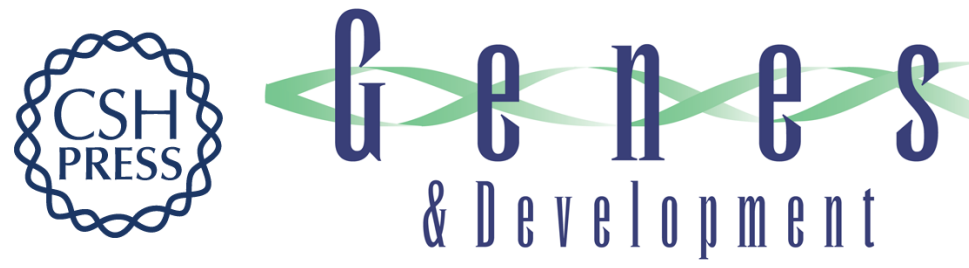

\section{Multiple functional domains of human transcription factor IIB: distinct interactions with two general transcription factors and RNA polymerase II.}

I Ha, S Roberts, E Maldonado, et al.

Genes Dev. 1993, 7:

Access the most recent version at doi:10.1101/gad.7.6.1021

References This article cites 32 articles, 12 of which can be accessed free at: http://genesdev.cshlp.org/content/7/6/1021.full.html\#ref-list-1

License

Email Alerting Service

Receive free email alerts when new articles cite this article - sign up in the box at the top right corner of the article or click here.

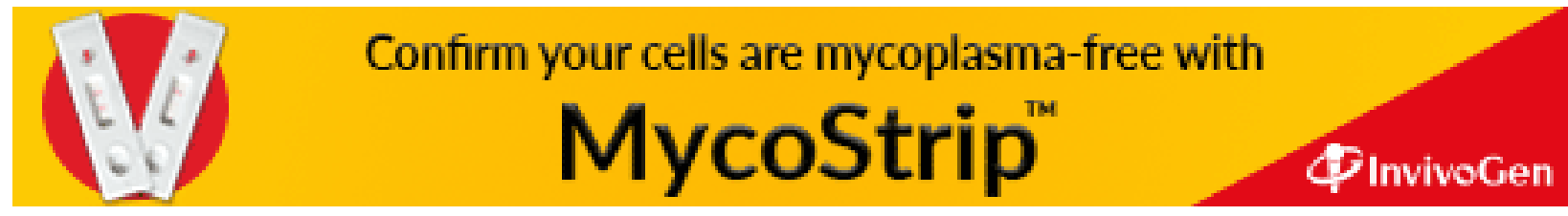

